\title{
ADVANTAGES FROM “DO-IT-YOURSELF” LOOPS AMONG CHILDREN AND ADOLESCENTS IN VARNA'S DIABETES CENTER
}

\author{
Yuliya Bazdarska ${ }^{1}$, Violeta Iotova ${ }^{1}$, Vilhelm Mladenov ${ }^{1}$, Veselin Boyadzhiev ${ }^{1}$, \\ Rositsa Stoycheva ${ }^{2}$, Sonya Galcheva ${ }^{1}$, Yana Bocheva ${ }^{3}$ \\ ${ }^{1}$ Department of Paediatrics, Faculty of Medicine, Medical University of Varna \\ ${ }^{2}$ Sector of Pediatrics, St. Marina Diagnostic and Consultation Center, \\ Medical University of Varna \\ ${ }^{3}$ Department of General Medicine and Clinical Laboratory, Faculty of Medicine, \\ Medical University of Varna
}

\begin{abstract}
INTRODUCTION: In the last 5 years, the use of automatic insulin delivery systems has been increasing among patients with type 1 diabetes mellitus (T1DM). It has been shown that they improve metabolic control, decrease the time spent in hypoglycemia and the number of episodes of nocturnal hypoglycemia; increase the time spent in target and are feasible and safe.
\end{abstract}

AIM: Our aim is to evaluate the glycemic control in children/adolescents using do-it-yourself (DIY) regulatory unapproved insulin-delivery loops vs sensor-augmented pump therapy (SAP).

MATERIALS AND METHODS: A total of 43 families with child/children with T1DM on pump treatment and continuous use of continuous glucose monitoring (CGM) were invited to participate in the study; 31 (72.1\%) of the families accepted. The children were followed for 6 months, a total of 196 patient months.

RESULTS: The study group consisted of 31 children with T1DM, 24 (77.4\%) of them were on SAP, and 7 (22.6\%) used DIY loops. No differences were observed in regard to age, duration of diabetes, and daily insulin dose between groups. Patients on DIY loops spent significantly more time in range ( 83.0 vs $68.8 \%, p=0.02$ ), less time in hyperglycemia above $14 \mathrm{mmol} / \mathrm{L}(2.1 \mathrm{vs} 8.6 \%, \mathrm{p}=0.02)$. They had significantly better HbAlc at the 6th month (6.5 vs $7.2 \%, p=0.006)$ vs SAP patients for the follow-up period. No severe hypoglycemia and diabetic ketoacidosis (DKA) occurred. There were no gender differences between and within both groups.

CONCLUSION: DIY unregistered loop systems showed promising results for better metabolic control at least in terms of mean blood glucose levels (BGLs) and without increasing the risk of severe hypoglycemia and DKA. Further impact e. g. mean insulin dose, long-term efficacy, consumed fat/protein in the

Address for correspondence:

Yuliya Bazdarska

Faculty of Medicine

Medical University of Varna

55 Marin Drinov St

9002 Varna

e-mail:yuliya.bazdarska@gmail.com daily diets, etc., remains to be studied in future larger and longer studies.

Keywords: T1DM, closed insulin-delivery loop, DIY, CGM, benefits 
Yuliya Bazdarska, Violeta Iotova, Vilhelm Mladenov et al.

\section{INTRODUCTION}

Automatic insulin delivery systems consist of: insulin pump (CSII), continuous glucose monitoring (CGM, sensor) and an algorithm that controls the required amount of the hormone. There are two types of loops: single hormone systems, which work only with fast-acting insulin analogues, and dual-hormone systems, which use insulin and glucagon. Sensor-augumented pump therapy (SAP) marks the beginning of the closing of the loop. The first step was the creation of the Minimed Paradigm Real Time System (insulin pump and sensor), which had the unique for that time function to stop automatically insulin infusion at a set in advance hypoglycemic value (1). Subsequent developments were based on predictive models that stop the supply of insulin before hypoglycaemia is reached and automatically restart insulin delivery with the rise of blood glucose (BG) up to pre-determined levels (Mimimed 640G with Smart Guard, Tandem Basal-IQ PLGS System) (2). Hybrid closed loop systems (HCLS) control, in automated mode, basal insulin rates according to the real time requirements. Additional boluses for the carbohydrate intake are still introduced manually. Quickly developing technological advances in the treatment of diabetes have led to the approval by FDA of two HCLS: MiniMed 670 G in 2017 and Tandem Control-IQ algorithm with an insulin pump t:slim X2 in $2019(3,4)$.

Progress in the development of automatic insulin systems is aimed at achieving optimal metabolic control, as it is known that good metabolic control reduces the long-term complications of type $1 \mathrm{di}$ abetes mellitus (T1DM) (5-8) as well as episodes of acute severe hypoglycaemia and diabetic ketoacidosis $(8,9)$. Despite the increasing introduction of new technologies and innovations in the treatment of T1DM (pumps, sensors, etc.), only one third of children and adolescents with T1DM in the U.S.A. meet the $\mathrm{HbAl}$ target of less than $7.5 \%(58 \mathrm{mmol} / \mathrm{mol})$ (10). Foster et al. published the latest results from the T1DM Exchange registry, which indicated that only $17 \%$ of patients achieved the goal (11). According to the last available report from the SWEET database, only $26.1 \%$ of children and adolescents cover the International Society for Pediatric and Adolescent Diabetes (ISPAD) criteria for HbAlc $<7.0 \%(<53 \mathrm{mmol} /$ mol) $(12,13)$. It is known that suboptimal diabetes control leads to dissatisfaction and increased burden among young people with T1DM, their families, and caregivers $(14,15)$. Recently published papers showed higher incidence of anger, depression, and other psychiatric disorders among adolescents with diabetes $(16,17)$. As a consequence, the quality of life among these age group and their families is lower $(18,19)$. The trend of the increasing incidence of T1DM by $3.4 \%$, annually in the pediatric population (20) and suboptimal metabolic control are the main prerequisites for the progress in automatic insulin delivery systems.

All known benefits of the hybrid closed loops in terms of metabolic control and reduction of blood glucose level (BGL) variability, as well as the reduced number of acute complications of T1DM, turn them into an innovative, efficacious and safe approach for treating children and adoslecents $(21,22)$.

The history of Do-it-yourself loop systems (DIYs) started with John Costik in 2013, the father of a child with a T1DM, who began to monitor remotely data from his son's sensor. He managed to download the data from the CGM to a local computer and forwarded it to the cloud. At the next level, Ben West, an engineer developing software for insulin pumps, managed to create an algorithm, which changed insulin infusion rates according to BG data from the sensor. He succeeded to "close the loop" and to codefine a platform called Open Source of Artificial Pancreas (OpenAPS, December 2014) $(23,24)$. DIY systems are HCLS, but have not been approved by the legal authorities. In order to ensure the safety of patients who use it in case of technical problems, when the algorithm stops working, the DIY system returns to basic basal-bolus regime of insulin pump therapy. Another difference, compared to HCLS, is that users themselves choose the brands of devices, which are connected in a closed loop system (pumps, CGM, smart phones and settings) $(25,26)$. The first results for the OpenAPS advantages were self-reported by users and showed a beneficial effect in terms of decreased HbAlc (from 7.1\% to 6.2\%), increased time in range (TIR) (from $58 \%$ to $81 \%$ ), improved sleep quality and satisfaction (27). The same team published in 2018 a retrospective analysis of data before and after initiation of DIY's and confirmed the benefits of the system even in patients with good control, and the time spent in hypoglycemia and the BGL variability 
were reduced $(26,27)$. At the best of the authors' current knowledge, only one scientific article about the benefits of OpenAPS in childhood is published by a Korean group (28).

\section{AIM}

The aim of the current study is to evaluate the glycemic control in children/adolescents using DIY's regulatory unapproved insulin-delivery loops vs sensor-augmented pump therapy (SAP).

\section{MATERIALS AND METHODS}

The study was conducted at the First Pediatric Clinic and CITAT-D (Center for Innovation Therapies and Advanced Technologies in Diabetes) at St. Marina University Hospital, Varna, Bulgaria for a 6-month period (196 patient/months of follow-up). All CITAT-D patients with T1DM from 0 to 18 years of age were invited to the study. All of them had used pump therapy with CGM for at least 6 months, which amounted to 43 . Out of the 43 contacted families, 31 (77.4\%) paricipants were included in the study after obtained parental consent for all the procedures. Educational sessions about the study protocol were delivered. Written instructions were provided to the families, including the minimal time of sensor usage ( $>70 \%$ for 10 to 14 days) before the scheduled visits (0-3-6 months). Participants were instructed to contact the team in case of technical problems with the devices. Recommendations were supplied about the place of insertion of the sensors according to the manufacturer's specification. Data from sensor devices were downloaded through the respective programs and saved in an electronic file (CVS and pdf) and/or paper version. Three types of sensors were used (FreeStyle Libre, Dexcom, EnLite 2.0). The data from the last 10-14 days before the day of the scheduled visit were analyzed. In the framework of the study, the following indicators were evaluated:

$\diamond$ Sensor usage (in \% of time);

$\diamond$ Time in range (TIR) - BGL 3.9-10 mmol/L;

$\diamond$ Time spent in hypoglycemia (BGL $<3.9$ $\mathrm{mmol} / \mathrm{L}$ );

$\diamond$ Time spent on significant hyperglycaemia (BGL $>14.0 \mathrm{mmol} / \mathrm{L}$ );

$\diamond$ Coefficient of variability of BGLs (CV\%).

Some of the internationally recommended parameters for assessment of metabolic control (time with $\mathrm{BG}<3.0 \mathrm{mmol} / \mathrm{L}$, BG $>10 \mathrm{mmol} / \mathrm{L}$, average BGLs and glucose management index) were not measured due to the technical obstacles linked to the lack of official registration of 2 of the sensors in our country.

All participants underwent a full clinical examination at baseline and at the end of the study ( $6^{\text {th }}$ month), with particular attention to the clinical data of acute and/or exacerbated chronic inflammatory processes, signs of growth and development derangements. On the day of the physical examinations, by protocol, participants came to the clinic in the morning at fast for the routine procedures: height and weight measurements, waist circumference, arterial pressure, insulin application sites and assessment of pubertal development (done by one investigator only). The mean daily insulin dose was determined from downloaded reports of the insulin pumps and/or the logs kept by the parents for the last 14 days before the visit.

\section{Sample Collection}

For the whole period of the study two fasting blood samples were taken at baseline and at the $6^{\text {th }}$ month for determing HbAlc. The parameter was determined by the reference immunobiodimetric inhibitory method in the central clinical laboratory of St. Marina University Hospital, Varna. During the study period, the laboratory participated in external and national laboratory control for HbAlc. The "cut off" of Hblc\% for good metabolic control until 2018 was $<7.5 \%$. After the 2018 publication of the ISPAD Clinical Consensus Statement, the target value was changed to $<7.0 \%$, which is routinely written in all patient documentation (patient file, discharge letters, outpatient visits records, etc.).

\section{Statistical Analysis}

Statistical analysis was performed using SPSS for Windows ver. 19. Descriptive analysis was applied. Logistic regression models were used to analyze correlations between TIR, time in hypoglycemia/hyperglycemia, HbAlc, and the type of the insulin therapy. Statistically significant differences were accepted if $\mathrm{p} \leq 0.05$.

\section{RESULTS}

Twenty-four (77.4\%) of all participants $(n=31)$ used SAP and the remaining seven (22.6\%) - DIYs 
(OpenAPS, Loop). No significant differences were observed between the two groups in terms of age, gender, daily insulin dose, and achieved control before CGM (Table 1). group $(4.5 \%$ vs $7.3 \%, \mathrm{p}=0.4)$. The time with $\mathrm{BGL}<3.5$ $\mathrm{mmol} / \mathrm{L}$ in both groups decreased during follow-up, predominantly for SAP users (from 3.5\% at baseline to $1.3 \%)$ (Fig. 3).

Table 1. Demographic characteristics of the participants The group of closed loops spent significantly more time in range compared to SAP. The trend was consistent throughout the entire observation period ( $82.3 \%$ vs $68 \%, p=0.0002)$. Increased TIR was more pronounced at the $3^{\text {rd }}$ and $6^{\text {th }}$ month for the follow-up period, which is demonstrated on Fig. 1.

\begin{tabular}{lccc} 
& SAP & DIY Loops & p \\
Age (y.) & $9.1 \pm 2.0$ & $10.1 \pm 2.0$ & NS \\
Gender (girls, \%) & $43 \%$ & $29 \%$ & NS \\
Duration T1D (y.) & $4.92 \pm 2.0$ & $5.03 \pm 1.7$ & NS \\
Insulin dose(U/kg/d) & & & \\
$\quad$ initial & $0.83 \pm 0.2$ & $0.76 \pm 0.2$ & NS \\
$\quad$ final & $0.75 \pm 0.2$ & $0.79 \pm 0.2$ & NS \\
HbAlc before CGM (\%) & $7.24 \pm 0.8$ & $6.97 \pm 1.2$ & NS \\
\hline
\end{tabular}

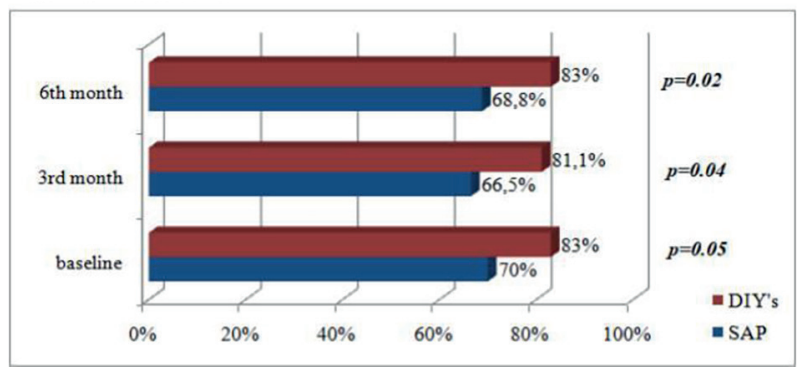

Fig. 1. Time in range trends

Participants on DIY systems spent significantly less time in hyperglycemia $>14 \mathrm{mmol} / \mathrm{L}$ (5.9 vs $8.7 \%, \mathrm{p}=0.02$ ) (Fig. 2).

Despite the lack of statistical significance, the same trend was observed for the time in hypoglycemia. In the DIY group, the average time spent with $\mathrm{BGL}<3.9 \mathrm{mmol} / \mathrm{L}$ was twice as low as that in the SAP

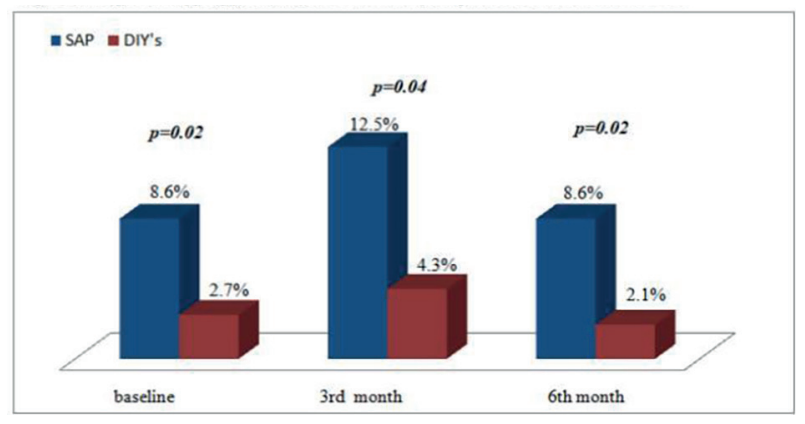

Fig. 2. Time spent in hyperglycemia above $14 \mathrm{mmol} / \mathrm{L}$. Comparison between SAP and DIYs

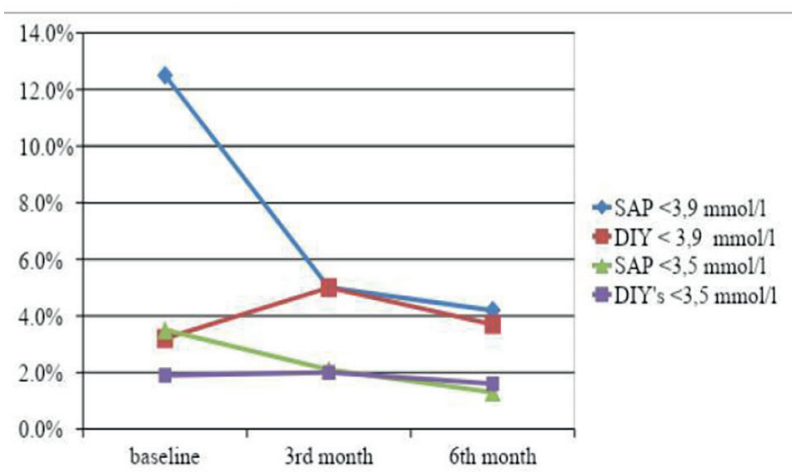

Fig. 3. Distribution of hypoglycemia

The DIY group achieved significantly better metabolic control compared to SAP: baseline HbAlc $-6.25 \pm 0.9 \%$ vs $7.2 \pm 0.9 \% \quad(\mathrm{p}<0.001)$ and $6^{\text {th }}$ month HbAlc - $6.5 \pm 0.4 \%$ vs $7.2 \pm 1.0 \%$ ( $\mathrm{p}=0.006)$, respectively.

In all subjects, the coefficient of variability $(\mathrm{CV})$ was below the upper limit of $36 \%$. No significant differences were found between groups: DIYs vs SAP baseline $25 \%$ vs $30 \%$; $6^{\text {th }}$ month $27 \%$ vs $27.5 \%$, NS.

\section{DISSCUSSION}

DIY's show promising results in achieving optimal diabetes care in children and adolescents. The evidence of advantages of the automatic insulin delivery systems from the current study are comparable to those published globally (23-28). The benefits of the closed loop systems in the treatment of T1DM 
in the studied group include optimal control, stable BGLs and reduced time spent in hypo- and hyperglycemia. The DIY group covers the latest global criteria for good diabetes control: CGM use $>70 \%$, TIR $>70 \%$, time with $\mathrm{BG}<3.9 \mathrm{mmol} / \mathrm{L}-<4.0 \%$, time in hyperglycemia $(>13.9 \mathrm{mmol} / \mathrm{L})<5 \%$, HbAlc $<7.0 \%$ $(13,29)$.

Initially, the majority of published studies on HCLS were conducted in camps, or in a hospital environment under direct 24-hour surveillance by medical staff with a small number of participants and a short-term follow-up. In a systematic review of the literature and meta-analysis of the currently available randomized controlled trials, most reported benefits of HCLS use are defined as TIR increased by $12.9 \%(9.02-16.16 \%, \mathrm{p}<0.0001)$ and episodes of hypoglycemia decreased by $2.45 \%$ ( -3.79 to $-1.11 \%$, $\mathrm{p}<0.0003)$ compared to SAP (21). The improvement in regard to $\mathrm{HbAlc} \%$ vary among different studies. Bergenstal et al. reported $\mathrm{HbAlc}$ reduction by $-0.5 \%$ (from 7.4\% to 6.4\%) for a 6-month follow-up in children and adults with T1DM started on closed loop (30). In a multricenter trial of closed loop, Garg et al. registered significantly lower HbAlc $(7.7 \% \pm 0.8 \%$ to $7.1 \% \pm 0.6 \%, \mathrm{p}<0.001)$. Key factors for achieving these results were increased TIR, lower mean BG levels, reduced time spent in hyperglycemia $>14 \mathrm{mmol} / \mathrm{L}$ (from $24.9 \% \pm 13.5 \%$ to $22.8 \% \pm 8.9 \%, \mathrm{p}=0.01$ ) and hypoglycemia (from $6.4 \% \pm 4.3 \%$ to $3.4 \% \pm 2.1 \%, \mathrm{p}<0.001$ ) (31).

In Korean children with T1DM with similar basal HbAlc levels, Choi et al. reported improved metabolic control among DIY users (6.8\% vs $6.3 \%$, $\mathrm{p}<0.001)$. In the current study, baseline HbA1c was slightly better than the $6^{\text {th }}$ month levels $(6.3 \%$ vs $6.5 \%, p>0.05)$ and no change in TIR was found at the 6th month. Limitation of the current study that may partly explain otherwise excellent results is the small number of participants with excellent initial metabolic control. Despite of the tight initial control of DIY group, time spent in hypoglycemia decreases further with $1.3 \%$ (from $5 \%$ to $3.7 \%$, NS) and time spent in hypeglycemia $>14 \mathrm{mmol} / \mathrm{L}$ dropped down by $0.6 \%(2.7 \%$ to $2.1 \%, \mathrm{NS})$. These results are similar to the reported by Italian and Korean studies (26-28).

One of the main barriers for optimal control in children and adolescents with T1DM is the fear of severe hypoglycemia (FOH), especially in families with a toddler. Some studies show that FOH is not always associated with an experienced episode $(32,33)$. Many trials have proven that HCLS significantly reduce the time spent below $3.9 \mathrm{mmol} / \mathrm{L}$, both at night and day time, regardless of the home or supervised conditions (22-23, 34-37). The safety and feasibility of HCLS in younger T1DM children (5-9 years) were demonstrated in a open-label, randomized, crossover study done by Del Favero et al. (37). The data showed that children using closed loops had a threefold reduction of time in hypoglycemia at the cost of higher mean BGLs and decreased TIR. Our data confirm less time spent below $3.9 \mathrm{mmol} / \mathrm{L}$ in the DIY group, especially at baseline, in comparison with SAP ( $5 \%$ vs $12.5 \%, \mathrm{p}=0.055)$, and no episodes of severe hypoglycemia occurred. We explain the improved time with $\mathrm{BG}<3.9 \mathrm{mmol} / \mathrm{L}$ in the SAP group (12.5\% vs $4.2 \%$, $\mathrm{p}=0.0001$ ) with the more intensive medical advice by email and strict follow-up during the study.

The latest data about the effects of chronic hyperglycemia and glucose variability shows association with reduced volumes of white and gray matter in brain regions involved in sensory-motor and cognitive functions (38). Also asymptomatic high BGLs lead to alterations in electrical brain activity (39). In this context, reduced time in hyperglycemia is as important as time below $3.9 \mathrm{mmol} / \mathrm{L}$. Most of the studies reported reducing time in hyperglycemia with different success, especially after the introduction of HCLS (33-34). Choi et al. published non-significant improvement in this parameter in children with T1DM (26-28, 30-31). In the follow-up period, the DIY group had threefold decrease in the time with hyperglycemia above $14 \mathrm{mmol} / \mathrm{L}$ compared to SAP. Glucose variability was stable for the entire observation period in the current study, and we did not find significant difference between both groups.

The disadvantages of most of the studies published so far are the small number of participants and the short observation periods. When we are discussing benefits of DIYs, in addition to the precise and smart algorithm, we should take into consideration the social and economic factors such as: parents' education, family environment, self-financing of treatment, which may lead to stricter adherence to the therapy and higher goals for the control explaining at least partially the excellent diabetes control results. 
Yuliya Bazdarska, Violeta Iotova, Vilhelm Mladenov et al.

\section{CONCLUSION}

DIY unregistered loop systems show promising results for better metabolic control without increasing the risk of severe hypoglycemia and diabetic ketoacidosis (DKA) at least in terms of mean BGLs. Further impact consists of consumed fat/protein, insulin dose, macro- and microvascular complications. Longer-term registry data and randomized studies are needed to further characterize the safety and efficacy of automatic insulin delivery systems. More research is needed to inform policy and practice in regard to DIY's.

\section{REFERENCES}

1. NCT01464346, Available from: https://www.accessdata.fda.gov/scripts/cdrh/cfdocs/cfpma/pma. cfm?id=P150019

2. Zhong A, Choudhary P, McMahon C, Agrawal P, Welsh J, Cordero T, et al. Effectiveness of automated insulin management features of the MiniMed ${ }^{\circledR}$ 640G Sensor-Augmented Insulin Pump Diabetes Technol Ther. 2016; 18(10):657-63. doi: 10.1089/ dia.2016.0216.

3. Available from: https://www.tandemdiabetes.com/ providers/products/basal-iq

4. Available from: https://www.accessdata.fda.gov/ cdrh_docs/pdf16/P160017S031B.pdf

5. Lifetime benefits and costs of intensive therapy as practiced in the diabetes control and complications trial. The Diabetes Control and Complications Trial Research Group. JAMA. 1996; 276(17):1409-15. doi: 10.1001/jama.1996.03540170053032.

6. Diabetes Control and Complications Trial/Epidemiology of Diabetes Interventions and Complications (DCCT/EDIC) Research Group1, Nathan DM, Zinman B, Cleary PA, Backlund JY, Genuth S, et al. Modern-day clinical course of type 1 diabetes mellitus after 30 years' duration: the diabetes control and complications trial/epidemiology of diabetes interventions and complications and Pittsburgh epidemiology of diabetes complications experience (1983-2005). Arch Intern Med. 2009; 169(14):130716. doi: 10.1001/archinternmed.2009.193.

7. Nordwall M, Abrahamsson M, Dhir M, Fredekison M, Ludvigsson J, Arngvist JH. Impact of HbAlc, followed from onset of type 1 diabetes, on the development of severe retinopathy and nephropathy: the VISS Study (Vascular Diabetic Complica- tions in Southeast Sweden). Diabetes Care. 2015; 38(2):308-15. doi: 10.2337/dc14-1203.

8. Misso ML, Egberts KJ, Page M, O’Connor D, Shaw J. Continuous subcutaneous insulin infusion (CSII) versus multiple insulin injections for type 1 diabetes mellitus. Cochrane Database Syst Rev. 2010; 1:CD005103. doi: 10.1002/14651858.CD005103.pub2.

9. Karges B, Schwandt A, Heidtmann B, Schierloh U, Binder E, Schierloh U, et al. Association of insulin pump therapy vs insulin injection therapy with severe hypoglycemia, ketoacidosis, and glycemic control among children, adolescents, and young adults with type 1 diabetes. JAMA. 2017; 318(14):1358-66. doi: 10.1001/jama.2017.13994.

10. Miller MK, Foster NC, Beck RW, Bergenstal RM, DuBose SN, DiMeglio LA, et al. Current state of type 1 diabetes treatment in the U.S.: updated data from the T1D Exchange clinic registry. Diabetes Care. 2015; 38(6):971-8. doi: 10.2337/dc15-0078.

11. Foster NC, Beck RW, Miller KM, Clements MA, Rickels MR, DiMeglio LA, et al. State of type 1 diabetes management and outcomes from the T1D Exchange in 2016-2018. Diabetes Technol Ther. 2019; 21(2):66-72. doi: 10.1089/dia.2018.0384.

12. SWEET Benchmarking Report 01/01/2019 31/07/2019, Available from: https:/www.sweetproject.org/benchmarking-reports.php.

13. ISPAD Guideline 2018, Available from: https:// www.ispad.org/page/ISPADGuidelines2018.

14. Weissberg-Benchell J, Antisdel-Lomaglio J. Diabetes-specific emotional distress among adolescents: feasibility, reliability, and validity of the problem areas in diabetes-teen version. Pediatr Diabetes. 2011; 12(4pt1):341-4. doi: 10.1111/j.1399-5448.2010.00720.x.

15. Hood KK, Beavers DP, Yi-Frazier J, Bell R, Dabelea D, Mckeown RE, et al. Psychosocial burden and glycemic control during the first 6 years of diabetes: results from the SEARCH for Diabetes in Youth study. J Adolesc Health. 2014; 55(4):498-504. doi: 10.1016/j.jadohealth.2014.03.011.

16. Buchberger B, Huppertz H, Krabbe L, Lux B, Mattivi JT, Siafarikas A. Symptoms of depression and anxiety in youth with type 1 diabetes: A systematic review and meta-analysis. Psychoneuroendocrinology. 2016; 70:70-84. doi: 10.1016/j. psyneuen.2016.04.019.

17. Cooper MN, Lin A, Alvares GA, de Klerk, NH, Jones, TW, Davis, EA. Psychiatric disorders dur- 
ing early adulthood in those with childhood onset type 1 diabetes: Rates and clinical risk factors from population-based follow-up. Pediatr Diabetes. 2017; 18(7):599-606. doi: 10.1111/pedi.12469.

18. Petersson C, Huus K, Enskär K, Hanberger L, Samulesson U, Åkesson K. Impact of type 1 diabetes on health-related quality of life among 8-18-yearold children. Compr Child Adolesc Nurs. 2016; 39(4):245-55. doi:10.1080/24694193.2016.1196265.

19. Sundberg F, Sand P, Forsander G. Health-related quality of life in preschool children with type $1 \mathrm{di}-$ abetes. Diabet Med. 2014; 32(1):116-9. doi: 10.1111/ dme.12557.

20. Patterson CC, Harjutsalo V, Resenbauer J, Neu A, Cinek O, Skrivarhaug T, et al. Trends and cyclical variation in the incidence of childhood type 1 diabetes in 26 European centres in the 25 year period 1989-2013: a multicentre prospective registration study. Diabetologia. 2019; 62(3):408-17. doi: 10.1007/ s00125-018-4763-3.

21. Weisman A, Bai JW, Cardinez M, Kramer CK, Perkins BA. Effect of artificial pancreas systems on glycaemic control in patients with type 1 diabetes: a systematic review and meta-analysis of outpatient randomised controlled trials. Lancet Diabetes Endocrinology. 2017; 5(7):501-12. doi: 10.1016/ s2213-8587(17)30167-5.

22. Musolino G, Dovc K, Boughton CK, Tauschmann M, Allen JM, Nagl K, et al. Reduced burden of diabetes and improved quality of life: Experiences from unrestricted day-and-night hybrid closedloop use in very young children with type $1 \mathrm{di}-$ abetes. Pediatr Diabetes. 2019; 20(6):794-9. doi: 10.1111/pedi.12872.

23. Lewis D. History and perspective on DIY closed looping. J Diabetes Sci Technol. 2019; 13(4):790-3. doi: $10.1177 / 1932296818808307$.

24. OpenAPS. Open APS. 2015. Available at: https:// OpenAPS.org.

25. Lewis D, Leibrand S, \#OpenAPS Community. Real-world use of Open Source Artificial Pancreas Systems. J Diabetes Sci Technol. 2016; 10(6):1411. doi:10.1177/1932296816665635.

26. Lewis DM, Swain RS, Donner TW. Improvements in A1C and time-in-range in DIY closed-loop (OpenAPS) users. Diabetes. 2018; 67(1):352-OR. doi: $10.2337 / \mathrm{db} 18-352-\mathrm{OR}$.

27. Provenzano V, Guastamacchia E, Brancato D, Cappiello G, Maioli A, Mancini R, et al. Closing the loop with OpenAPS in people with type 1 diabetesexperience from Italy. Diabetes. 2018; 67(1):993-P. doi: 10.2337/db18-993-P.

28. Choi SB, Hong ES, Noh YH. Open artificial pancreas system reduced hypoglycemia and improved glycemic control in patients with type $1 \mathrm{di}-$ abetes. Diabetes. 2018; 67(1):964-P. doi: 10.2337/ db18-964-P.

29. Battelino T, Danne T, Bergenstal RM, Amiel SA, Beck R, Biester T, et al. Clinical targets for continuous glucose monitoring data interpretation: recommendations From the International Consensus on Time in Range. Diabetes Care. 2019; 42(8):1593603. doi: 10.2337/dci19-0028.

30. Bergenstal RM, Garg S, Weinzimer SA, Buckingham BA, Bode BW, Tamborlane WV, et al. Safety of a hybrid closed-loop insulin delivery system in patients with type 1 diabetes. JAMA. 2016; 316(13):1407-8. doi:10.1001/jama.2016.11708

31. Garg SK, Weinzimer SA, Tamborlane WV, Buckingham BA, Bode BW, Bailey TS, et al. Glucose outcomes with the in-home use of a hybrid closed-loop insulin delivery system in adolescents and adults with type 1 diabetes. Diabetes Technol Ther. 2017; 19(3):155-63. doi: 10.1089/dia.2016.0421.

32. Patton SR, Dolan LM, Henry R, Powers SW. Fear of hypoglycemia in parents of young children with type 1 diabetes mellitus. J Clin Psychol Med Settings. 2008; 15(3):252-9. doi: 10.1007/ s10880-008-9123-X.

33. Gonder-Frederick LA, Fisher CD, Ritterband LM, Cox DJ, Hou L, DasGupta AA et al. Predictors of fear of hypoglycemia in adolescents with type $1 \mathrm{di}-$ abetes and their parents. Pediatr Diabetes. 2006; 7(4):215-22. doi: 10.1111/j.1399-5448.2006.00182.x.

34. Hovorka R, Kumareswaran K, Harris J, Allen JM, Elleri D, Xing D, et al. Overnight closed loop insulin delivery (artificial pancreas) in adults with type 1 diabetes: crossover randomised controlled studies. BMJ. 2011; 342:d1855. doi: 10.1136/bmj.d1855.

35. Ly TT, Keenan DB, Roy A, Han J, Grosman B, Cantwell $\mathrm{M}$ et al. Automated Overnight ClosedLoop Control Using a Proportional-Integral-Derivative Algorithm with Insulin Feedback in Children and Adolescents with Type 1 Diabetes at Diabetes Camp. Diabetes Technology \& Therapeutics 2016; 18(6):377-384.

36. Phillip M, Battelino T, Atlas E, Kordonouri O, Bratina N, Miller S, et al. Nocturnal glucose con- 
Yuliya Bazdarska, Violeta Iotova, Vilhelm Mladenov et al.

trol with an artificial pancreas at a diabetes camp. N Engl J Med. 2013; 368(9):824-33. doi: 10.1056/ nejmoa1206881.

37. Del Favero S, Boscari F, Messori M, Rabbone I, Bonfanti R, Sabbion A, et al. Randomized summer camp crossover trial in 5- to 9-year-old children: outpatient wearable artificial pancreas is feasible and safe. Diabetes Care 2016; 39(7):1180-5. doi: 10.2337/dc15-2815.

38. Mauras N, Mazaika P, Buckingham B, Weinzimer S, White NH, Tsalikaian E, et al. Longitudinal assessment of neuroanatomical and cognitive differences in young children with type 1 diabetes: association with hyperglycemia. Diabetes. 2015; 64(5):1770-9. doi: 10.2337/db14-1445.
39. Rachmiel M, Cohen M, Heymen E, Lezinger M, Inbar D, Gilat S, et al. Hyperglycemia is associated with simultaneous alterations in electrical brain activity in youths with type 1 diabetes mellitus. Clin Neurophysiol. 2016; 127(2):1188-95. doi: 10.1016/j. clinph.2015.07.011. 